\title{
COMMENTARY
}

\section{Matters of Life and Death}

\author{
Andrea Sikora Newsome, PharmD, ${ }^{\mathrm{a}, \mathrm{b}}$ Brian Murray, PharmD ${ }^{\mathrm{c}}$ \\ ${ }^{\text {a } U n i v e r s i t y ~ o f ~ G e o r g i a, ~ C o l l e g e ~ o f ~ P h a r m a c y, ~ A u g u s t a, ~ G e o r g i a ~}$ \\ ${ }^{\mathrm{b}}$ Augusta University Medical Center, Augusta, Georgia \\ ${ }^{\mathrm{c}}$ University of North Carolina Medical Center, Chapel Hill, North Carolina
}

Correspondence: Andrea Sikora Newsome, University of Georgia, College of Pharmacy, 120 15th St, HM-118, Augusta, GA 30912. Tel: 706-721-3826. Email: sikora@uga.edu

Submitted March 11, 2021; accepted June 20, 2021; ePublished July 2021

Keywords: critical care, education, end of life

Wherever the art of Medicine is loved, there is also a love of Humanity. - Hippocrates

Death is the common denominator of the human, and therefore health care, experience. The advent of modern medicine has brought significant changes to how and where we die. Compared to just a hundred years ago when a majority of individuals died at home, an exceptional 30\% of Americans will die in an intensive care unit (ICU) with end of life costs exceeding $\$ 200$ billion annually. ${ }^{1,2}$ Therefore, to practice in critical care (or any discipline of medicine) brings us as clinicians and as educators in touch with death and dying. This reality is especially true in the current COVID-19 pandemic, during which clinicians are experiencing death in unprecedented volume and under especially traumatic circumstances. ${ }^{3,4}$ Unpredictable clinical courses, sudden decompensations, and the absence of family members and loved ones as patients suffer and die alone have become the hallmarks of ICU care. Without the necessary support and coping skills, these experiences may lead to feelings of guilt, resentment, and regret that affect student and resident clinicians in unseen ways, ranging from burnout to depression and post-traumatic stress. ${ }^{5-7}$ Beyond the direct consequences for clinicians and trainees, these effects can have meaningful ramifications for patient safety. ${ }^{8}$ Here, we posit that end-of-life discussions should be foundational to any ICU learning experience and propose a model for these discussions.

Over the last decade, the profession of pharmacy has placed significant emphasis on involvement of the pharmacist in direct patient care. ${ }^{9}$ This emphasis more consistently positions a pharmacist at the bedside, where they are involved in interprofessional decision making as the pharmacotherapy expert. While the presence of the bedside pharmacist has increased dramatically, associated curricular changes have been slower to develop, especially in the discipline of critical care. In 1987, 38\% of pharmacy programs reported offering no education related to death and dying or end-of-life care, as opposed to only $4 \%$ and $5 \%$ of medical and nursing programs, respectively. ${ }^{10}$ While these statistics have improved, as of 2012, 20\% of pharmacy programs surveyed still lacked any formal preparation for end-of-life care, and programs that do offer this training have an average of under 7 hours throughout the entire curriculum. ${ }^{11}$ These findings are despite the Accreditation Council for Pharmacy Education (ACPE) accreditation standards that focus on providing "patient-centered collaborative care" across the patient's lifespan. ${ }^{12}$ Notably, residency training standards have no recommendations regarding this topic. As such, the reality is that pharmacy trainees will face death and related events in their clinical experiences and post-graduate training, and for many, it will be their first experience with death and dying not only as a health care provider but potentially overall. ${ }^{13}$

First experiences with death are often the most memorable and emotional, even for students who do not have a strong connection to a particular patient. ${ }^{14}$ In addition, most pharmacy residents are not emotionally prepared to face endof-life situations and other highly emotional experiences without appropriate guidance and support. ${ }^{15}$ Including more endof-life exposure and training in the pre-clinical curriculum may alleviate some of the stressors associated with death and dying; indeed, classroom interventions have been shown to impact empathy and student perceptions of death and dying, ${ }^{16,17}$ and hands-on simulations may be even more effective in preparing students for end-of-life scenarios. ${ }^{13,18-19}$ However, evidence suggests that pre-clinical classroom preparation may not have a long-term impact. ${ }^{20}$ For this reason, the role of preceptors, program directors, peers, and institutional culture in the clinical environment is paramount for developing practice-ready pharmacists with effective coping skills capable of reconciling empathy and professionalism in 
end-of-life settings. It is imperative that preceptors have the requisite skills and comfort in having crucial conversations with students and residents surrounding end-of-life care.

The support and guidance that students and residents do receive mostly takes the form of informal one-on-one discussions with preceptors and residency program directors. ${ }^{15}$ Given prevailing trends in the United States health care system and rotational requirements, most pharmacy students and residents will have their first exposure to end-of-life care in an ICU setting. Unfortunately, the effects of working in an ICU environment are manifold and include that providers have significantly higher rates of burnout, which has ramifications both for adverse patient outcomes and individual wellness. ${ }^{21}$ This burnout and resulting emotional detachment is reflected in the "hidden curriculum" (or the values, attitudes, beliefs, and behaviors that students learn through their daily interaction with health care providers and how they learn what the medical community thinks is important to being a doctor ${ }^{14}$ ) that learners are exposed to in ICU practice areas. Pervasive burnout and emotional exhaustion may affect the way that superiors and role models respond to end-oflife situations, while also making it more difficult for them to sympathize and connect with learners having a "new" experience with death. This culture may lead to a perceived lack of support at a time that is critical for shaping the future attitudes, emotional responses, and coping skills of learners. Indeed, while emotional stoicism is often prized in ICU culture, this coping strategy is counterproductive and can lead to an internal tension for students and residents struggling to reconcile the need to have empathy for their patients with the perceived need for emotional detachment from traumatic situations. ${ }^{14,22-25}$ The ability for a preceptor to guide learners through meaningful discussions and to effectively manage these complex and often difficult scenarios involving end-of-life is an essential skill and can add great meaning to an experiential rotation. ${ }^{26}$ Without these discussions and appropriate modeling, highly emotional experiences often lead to the adoption of coping strategies that are detrimental to long-term well-being and a conflicted professional identity. Table 1 provides a brief summary of some suggested background resources to support these discussions.

Building upon Wilsey's call for de-brief discussions, Ku beautifully summarizes how to handle these sessions and model empathetic communication skills. ${ }^{27-28}$ She emphasizes "timely and consistent debriefing" with three core components: (1) model grief and emotional response, (2) focus on the emotional aspects of the death and dying process rather than just the medical or scientific aspects of the patient case, and (3) discuss strategies and resources for coping with grief; however, we emphasize going beyond these more reactionary discussions to a proactive and structured approach. Here, preceptors would set aside not only time for debrief discussions but normalize and standardize this topic for every learning experience. Although this topic must be adapted to the specific nature of the rotational experience and the learner, we proposed a construct that entails establishing overall perspective with the learner (eg, epidemiology and historical viewpoints of end of life), summarizing pharmacotherapeutic goals and strategies in line with "I will use those regimens which will benefit my patients according to my greatest ability and judgment, and I will do no harm or injustice to them," and finally allowing for time to de-brief from the emotionally taxing environment of the ICU and open discussion of effective coping strategies that maximize wellness and minimize burnout. While limited data are available regarding optimal strategies for educating pharmacy learners on this important topic, we have provided suggested learning objectives and activities for these discussions in Table 2.

Further, integrating concepts of palliative care and associated pharmacotherapeutic management into these endof-life discussions can provide valuable perspective. The goal of palliative care is to "maintain and improve the quality of life of all patients and their families during any stage of life-threatening illness" by aiming to "prevent and relieve suffering by early identification, assessment, and treatment of physical and psychological symptoms, as well as emotional, and spiritual distress." ${ }^{29}$ As such, intensive care medicine and palliative care are ideally integrated and complementary approaches with benefits for patients, caregivers, and critical care clinicians alike. ${ }^{30}$ Notably, palliative care is associated with higher ratings of quality of life, death, and end-of-life care in addition to greater wellbeing for bereaved individuals. ${ }^{31-32}$ Incorporating these elements can underscore the important role of the pharmacist in end-of-life and palliative care, both with optimizing pharmacotherapy and developing end-of-life protocols. ${ }^{33,34}$

Overall, patient-centered management of end-of-life is a necessary competency for graduating clinicians. Educators and preceptors can guide their trainees through this topic using a combination of discussion-oriented knowledge transfer, empathetic listening and deliberate debriefing, and provision of further resources. Indeed, taking time to have these vital end-of-life discussions may serve to help educators model Hippocrates when he states "most especially must I tread with care in matters of life and death."

\section{ACKNOWLEDGMENTS}

Conflicts of Interest: Dr. Newsome has received research funding through the National Center for Advancing Translational Sciences (NCATS) of the National Institutes of Health (NIH) under Award Numbers UL1TR002378 and KL2TR002381. 


\section{REFERENCES}

1. Rothman DJ. Where we die. N Engl J Med 2014;370:2457-60.

2. Luce JM, Rubenfeld GD. Can health care costs be reduced by limiting intensive care at the end of life? Am J Respir Crit Care Med. 2002;165:750-754.

3. Wakam GK, Montgomery JR, Biesterveld BE, Brown CS. Not dying alone - mondern compassionate care in the COVID-19 pandemic. $N$ Engl J Med. 2020;382(24):e88.

4. Rosenbaum L. Harnessing our humanity - how Washington's health care workers have risen to the pandemic challenge. N Engl J Med. 2020;382(22):2069-2071.

5. Leiter RE. Reentry. N Engl J Med. 2020;383(27):e141.

6. Shapiro J, McDonald TB. Supporting clinicians during COVID-19 and beyond - learning from past failures and envisioining new strategies. $N$ Engl J Med. 2020;383(27):e142.

7. DeForest A. The new stability. N Engl J Med. 2020;383(18):1708-1709.

8. Garcia CL, et al. Influence of burnout on patient safety: systematic review and meta-analysis. Medicina (Kaunas). 2019;30(55):553.

9. American Society of Health-System Pharmacists. The consensus of the Pharmacy Practice Model Summit. Am J Health-Syst Pharm. 2011;68:1146-1152.

10. Dickinson GE, Sumner ED, Durand RP. Death education in US professional colleges: medical, nursing, and pharmacy. Death Stud. 1987;11(1):57-61.

11. Dickinson GE. End-of-life and palliative care education in US pharmacy schools. Am J Hosp Palliat Care. 2013;30(6):532-535.

12. ACPE Accreditation Standards. https://www.acpe-accredit.org/pharmd-program-accreditation/. (accessed 6 March 2021).

13. Gilliland I, Frei BL, McNeill J, Stovall J. Use of high-fidelity simulation to teach end-of-life care to pharmacy students in an interdisciplinary course. Am J Pharm Educ. 2012;76(4):66.

14. Rhodes-Kropf J, Carmody SS, Seltzer D, Redinbaugh E, Gadmer N, Block SD, Arnold RM. "This is just too awful; I can't believe I experienced that...": medical students' reactions to their "most memorable" patient death. Acad Med. 2005;80(7):634-640.

15. Pileggi DJ, Fugit A, Romanelli F, Winstead PS, Lawson A, Deep KS, Cook AM. Pharmacy residents' preparedness for the emotional challenges of patient care. Am J Health Syst Pharm. 2015;72(17):1475-1480.

16. Manolakis ML, Olin JL, Thornton PL, Dolder CR, Hanrahan C. A module on death and dying to develop empathy in student pharmacists. Am J Pharm Educ. 2011;75(4):71.

17. Beall JW, Broeseker AE. Pharmacy students' attitudes towards death and end-of-life care. Am J Pharm Educ. 2010;74(6): 104.

18. Gannon J, Motycka C, Egelund E, Kraemer DF, Smith WT, Solomon K. Teachign end-of-life care using interprofessional simulation. J Nurs Educ. 2017;56(4):205-210.

19. Egelund EF, Gannon J, Motycka C, Smith WT, Kraemer DF, Solomon KH. A simulated approach to fostering competency in end-of-life care among pharmacy students. Am J Pharm Educ. 2019;83(4):6904.

20. Lor KB, Truong JT, IP EJ, Barnett MJ. A randomized prospective study on outcomes of an empathy intervention among second-year student pharmacists. Am J Pharm Educ. 2015;79(2):18.

21. Kerlin MP, McPeake J, Mikkelsen ME. Burnout and Joy in the Profession of Critical Care Medicine. Crit Care. 2020;24:98.

22. Kelly E, Nisker J. Medical students' first clinical exerperiences of death. Med Educ. 2010;44(4):421-8.

23. Trivate T, Dennis AA, Sholl S, Wilkinson T. Learning and coping through reflection: exploring patient death experiences of medical students. BMC Med Educ. 2019;19(1):451.

24. Ho CY, Kow CS, Chia CHJ, et al. The impact of death and dying on the personhood of medical students: a systematic scoping review. BMC Med Educ. 2020;20(1):516.

25. Smith-Han K, Martyn H, Barrett A, Nicholson H. "That's not what you expect to do as a doctor, you know, you don't expect your patients to die." Death as a learning experience for undergraduate medical students. BMC Med Educ. 2016;16:108.

26. Anderson WG, Williams JE, Bost JE, Barnard D. Exposure to death is associated with positive attitudes and higher knowledge about end-of-life care in graduating medical students. J Palliat Med. 2008;11:1227-33.

27. Wilsey, HA. An Important lesson: Dealing with Death. Currents in Pharmacy Teaching and Learning. 2019. https://cptlpulses.com/2019/02/19/dealingwithdeath/ (accessed 2021 January 8). 
28. Ku, J. Pausing to Reflect and Debrief: Emotional Processing in the Face of Death. Currents in Pharmacy Teaching and Learning. 2019. https://cptlpulses.com/2019/05/28/pausing-to-reflect-and-debrief-emotional-processing-in-theface-of-death/ (accessed 2021 January 8).

29. World Health Association. 2021. WHO Definition of Palliative Care. http://www.who.int/cancer/palliative/definition/en (accessed 2021 May 11).

30. Cook D, Rocker G. Dying with dignity in the intensive care unit. N Engl J Med. 2014 Jun 26;370(26):2506-2514.

31. Mercadante S, Gregoretti C, Coregiani A. Palliative care in intensive care units: why, where, what, who, when, how. BMC Anesthesiol. 2018; 18: 106.

32. Aslakson RA, Curtis RJ, Nelson JE. The changing role of palliative care in the ICU. Crit Care Med. 2014 Nov;42(11):2418-2428.

33. Walker KA, Scarpaci L, McPherson ML. Fifty reasons to love your palliative care pharmacist. Am J Hosp Palliat Care. 2010 Dec;27(8):511-513.

34. Herndon CM, Nee D, Atayee RS, et al. ASHP guidelines on the pharmacist's role in palliative and hospice care. Am J Health Syst Pharm. 2016 Sep;73(17):1351-1367. 
Table 1. Summary of Key Resources

Reference Summary

Books

1. Being Mortal by Atul Gawande

Surgeon, writer, and public health researcher discusses his experience both of his dying father and managing end-of-life in his medical career

\section{Modern Death by Haider Warraich}

3. In Shock by Rana Awdish

4. People Like that are the Only People Here: Canonical Babbling in Peed Oink by Lorrie Moore

Perspective Pieces

5. Gawande A. Letting Go. The New Yorker. 2 August 2010.

6. Ryon DL. The rime of the ancient intensivist. Critical Care Medicine. April 1995 - Volume 23 - Issue 4 - p 773 - 774.

7. Barbash IJ. Silent Space. JAMA 2018;320:1105-6.

8. Reeder-Hayes KE. Haunted. J Clin Oncol 2017;35:113-4.

9. Gupta A. Harry Potter, Magic, and Medicine. JAMA Intern Med 2018;178:747-8.
Physician explores how modern medicine has impacted the universal truth of death and dying

Physician recounts her near-death and related intensive care unit experience as well as recovery providing a vital patient oriented perspective

Poignant short story recounting the parental perspective of their child being cared for in the pediatric oncology ward

Basis of the book 'Being Mortal,' this essay brilliantly recounts the journey of a terminal oncology patient as well as end of life care

This moving poem recounts the critical care clinician's experience of a dying patient and has also been published multiple times since its original release

Discusses the value of pausing and reflecting following the death of a patient as part of the grief (and wellness) process for providers

Oncologist reflects upon the years of patient care, beautifully acknowledging how the stories (and deaths) of patients have shaped her

Physician reflection on the role of the provider when 'nothing can be done' and the art of being present and good listening

Review and discussion of the sky-rocketing healthcare costs at the end-of-life in the United States that provides important perspective

Provides important introduction to effective listening practices for difficult discussions and crucial conversations emotion-respecting. Updated 2018. Accessed March 28, 2019.
10. VitalTalk. Responding to Emotion: Respecting.
VitalkTalk. https://www.vitaltalk.org/guides/responding-to-

9. Luce JM, Rubenfeld GD. Can health care costs be reduced by limiting intensive care at the end of life? Am J Respir Crit Care Med 2002;165:750-4.

Palliative care and pharmacotherapy

11. Clary PL, Lawson P. Pharmacologic pearls for end-of-life care. Am Fam Physician 2009;79:1059-65.

12. Ross DD, Alexander CS. Management of common symptoms in terminally ill patients: Part I. Fatigue, anorexia, cachexia, nausea and vomiting. Am Fam Physician 2001;64:807-14.

13. Ross DD, Alexander CS. Management of common symptoms in terminally ill patients: Part II. Constipation,
Introductory review for medication management

Expanded discussion of end-of-life pharmacotherapy management that orients the reader to a different perspective with regard to pharmacotherapeutic care

Second installment on end-of-life pharmacotherapy management focusing on 
delirium and dyspnea. Am Fam Physician 2001;64:1019-26.

14. Cook D, Rocker G. Dying with dignity in the intensive care unit. N Engl J Med. 2014 Jun 26;370(26):2506-14. common issues experiences by patients

Thoughtful and practice guide to palliative care best practices regarding caring for patients and families in the dying process as it occurs in the ICU

Table 2. Learning Objectives and Activities for an End-of-Life Discussion

Learning Objective

Trace relevant epidemiology, outcomes, and historical perspectives regarding end of life care, with a focus on the ICU setting

Summarize goals of palliative care and pharmacotherapeutic management strategies during end-of-life care
Associated Activity

Discuss prognosis of cardiac arrest / acute

cardiovascular life support interventions

Review mortality risk predictions in the ICU setting

Discuss the shift of goals from 'prolonging life' to 'providing comfort' and how this may be different than other rotations and experiences

Examine common misconceptions about palliative care in the ICU (eg, that palliative care hastens death, that ICU care/palliative care are opposing processes)

Review pharmacokinetics and practicalities of end of life medication dosing

Provide discussion of code blue activities and pharmacist participation

Discuss rotation specific end of life experiences and explore learner attitudes and beliefs regarding their role as a clinician in providing end of life care

Conduct de-brief discussions regarding patient specific experiences are recommended

Adopt an open attitude and creation of an emotionally safe environment for expression of emotions, beliefs, experiences, values, questions, etc.

Practice empathetic communication skills (eg, active listening, nonverbal expressions, etc.)

Cultivate effective coping strategies for managing Complete discussion in the first half of the rotation or other ICU learning experience

Discuss personal experiences or rituals as part of preceptor role-modeling 